\title{
A Comparison of Gaze-Based and Gesture-Based Input for a Point-and-Click Task
}

\author{
Dominic Canare \\ Faculty: Barbara Chaparro, Jibo He \\ Department of Psychology, Fairmount College of Liberal Arts and Sciences
}

\begin{abstract}
Alternative input devices to the computer mouse are becoming more affordable and accessible. With greater availability, they have the potential to provide greater access to information for more users in more environments. However, most user interfaces are built to be mouse-driven, and the adoption of these new technologies may depend on their ability to work with these existing interfaces. This study examined performance with gesture-control and gaze-tracking devices and compared them to a traditional mouse for a standard Fitts' Law point-and-click task. Participants were capable of using both gesture-controlled and gaze-tracking devices to control an on-screen keyboard quickly and accurately enough to complete the majority of trials. However, both devices were significantly slower than the mouse, and were more prone to missclicks and failures. Our subjective measures indicate that participants found these alternative input devices to be more taxing than the familiar mouse.

In order to make effective use of these devices, researchers, designers, and developers must find control schemes which take advantage of the alternative devices' benefits while curtailing the drawbacks. While this study does show that these alternative input devices can be used to control a computer's cursor in a point-and-click environment, it is inefficient, error-prone, and difficult for the user.
\end{abstract}

